\title{
Transport of superradiant excitons in GaAs single quantum wells
}

\author{
P. Vledder, A. V. Akimov, * and J. I. Dijkhuis \\ Faculty of Physics and Astronomy, and Debye Institute, University of Utrecht, P.O. Box 80.000, 3508 TA Utrecht, The Netherlands \\ J. Kusano, Y. Aoyagi, and T. Sugano \\ Frontier Research Program, The Institute of Physical and Chemical Research, RIKEN, Wako, Saitama 351-01, Japan
}

(Received 16 July 1997)

\begin{abstract}
The dynamics of resonantly excited $1 s-H H$ excitons in GaAs single quantum wells is studied by picosecond transient reflection and photon echo. The energy relaxation and transport properties of the excitons are probed, making use of the surface electric field. At low exciton densities and $5 \mathrm{~K}$, transport appears to be absent, and superradiant decay takes the place of free excitons with characteristic times ranging from 5 to $13 \mathrm{ps}$, depending on the well width. The polarization decay is governed by energy relaxation, and a coherence length is found for these free excitons of $\sim 200 \mathrm{~nm}$. Low in the exciton resonance, we measure a much slower radiative decay and smaller coherence lengths, due to localization. Here both radiative decay and pure dephasing contribute to the polarization dephasing rate. At higher exciton densities and temperature, transport is observed, induced by exciton-exciton and exciton-phonon collisions. For free excitons it is demonstrated that the increased polarization dephasing rate is exclusively due to exciton transport, and not to pure dephasing. For localized excitons, pure dephasing processes prevail in $T_{2}$, and thermally activated transport is found. [S0163-1829(97)05847-5]
\end{abstract}

\section{INTRODUCTION}

The radiative recombination of excitons in two dimensions is fundamentally different from the three-dimensional case. In bulk, the strong coupling between light and excitons gives rise to an interesting eigenstate, the polariton. Radiative decay, then, requires phonons or other translationalinvariance breaking entities such as defects, impurities or interfaces. Quantum wells (QW's), however, inherently break translational invariance in one direction. As a consequence, a radiative decay channel opens up, at least for excitons with a center-of-mass in-plane wave number $q_{\|}$ smaller than $\pi / \lambda$, with $\lambda=\lambda_{0} / n, \lambda_{0}$ the in vacuo wavelength of the photon, and $n$ the index of refraction. The small-wave-number excitons have a giant dipole moment since they are a linear superposition of a large number of electron-hole wave functions, and exhibit Dicke superradiance. ${ }^{1}$ This phenomenon is well known in atomic physics. It refers to the situation in which $N$ identically prepared two-level atoms radiate from a region smaller than the optical wavelength. Their collective radiative decay rate is enhanced with a factor $N$ relative to that of a single atom. The exciton wave function is also collective in the sense that it represents a coherent superposition of a large number of electron-hole states. Superradiance has been discussed by numerous authors, both for one-dimensional (1D) Frenkel excitons in disordered aggregates 2,3 and 2D Wannier excitons in semiconductor thin films. ${ }^{4-9}$

Hanamura ${ }^{7}$ calculated the radiative decay rate $\Gamma_{0}$ of free 2D excitons with a zero center-of-mass momentum:

$$
\Gamma_{0}=1 / \tau_{0}=24 \pi\left(\frac{\lambda}{a_{B}}\right)^{2} \gamma_{s} .
$$

Here $\gamma_{s}=45\left|\mathbf{d}_{\mathrm{cv}}\right|^{2} / 3 \hbar \lambda^{3}$, with $\mathbf{d}_{\mathrm{cv}}$ the dipole matrix element, represents the one-electron radiative decay rate. One can un- derstand the factor $\left(\lambda / a_{B}\right)^{2}$ in Eq. (1) qualitatively: the radiative decay is enhanced with respect to the one-electron case by a factor equal to the number of coherent oscillators within the area of $\lambda^{2}$. In a $100-\AA \mathrm{GaAs}$ single quantum well (SQW) $\tau_{0}=9.9 \mathrm{ps,} \mathrm{according} \mathrm{to} \mathrm{Eq.} \mathrm{(1).} \mathrm{Later}$ calculations, ${ }^{8,9}$ however, give values for $\tau_{0}$ that are a factor of 3 higher.

In these calculations, the excitons are considered to be ideal, i.e., of infinite extension, and thus radiate in a directional way in exactly one single photon mode. In reality, however, excitons have a limited coherence length $L_{c}$ that, if short enough, increases the radiative lifetime. Indeed, if $L_{c}$ $<\lambda$, the superradiant effect becomes less prominent. The coherence length $L_{c}$ appears to be directly related to the pure dephasing time $T_{2}^{\prime}$ of an exciton, ${ }^{10}$

$$
L_{c}=\sqrt{\hbar T_{2}^{\prime} / M}
$$

with the in-plane exciton mass $M=0.27 m_{0}$, and $m_{0}$ the electron mass. The radiative decay rate for excitons in the limit of $L_{c}<\lambda$ reads $^{10}$

$$
\Gamma^{\prime}=24 \pi\left(\frac{2 L_{c}}{a_{B}}\right)^{2} \gamma_{s}
$$

There are a number of experiments that measure the lifetime of $1 s-H H$ excitons in GaAs QW's, mostly using the technique of time-resolved luminescence. For a $45-\AA$ SQW, Deveaud et al. ${ }^{11}$ deduced a value for the exciton lifetime of $10 \pm 4 \mathrm{ps}$, based on resonantly excited exciton (REE) luminescence experiments and measurements of the resonance line profile. Vinatierri et al. ${ }^{12}$ obtained the radiative lifetime of excitons to be $40 \mathrm{ps}$ in a 15 -period GaAs $150-\AA$ multiplequantum-well (MQW) sample with $150-\AA \mathrm{Al}_{x} \mathrm{Ga}_{1-x} \mathrm{As}$ barriers. They combined a measurement of the luminescent lifetime with that of the homogeneous linewidth determined by 
four-wave mixing (FWM). Recently a FWM study on a SQW and MQW Bragg structures ${ }^{13}$ has indicated that the exciton lifetime should be $<10 \mathrm{ps}$. Wang et al. ${ }^{14}$ measured a 8-ps lifetime in a 15 -period $175-\AA \mathrm{MQW}$ with 150 - $\AA$ barriers.

Transport properties of REE in real structures appear to be dependent on the excitation position in the inhomogeneously broadened line. Hegarty and Sturge ${ }^{15}$ showed that a mobility edge exists below which excitons are considered to be immobile. Excitons with energies higher than the mobility edge show diffusive transport with a diffusion coefficient $D \sim 10 \mathrm{~cm}^{2} \mathrm{~s}^{-1} .{ }^{15}$ In the last decade transport of resonantly excited excitons was studied by different techniques. Hillmer et al. ${ }^{16}$ measured the lateral transport of resonantly and nonresonantly excited 2D excitons, using a time-of-flight method. The resonantly created excitons were shown to radiate from the island where they are created. Deveaud et al. ${ }^{17}$ demonstrated in a time-resolved luminescence experiment that nonresonantly excited excitons move from a region of $N$ monolayers to a region with $N+1$ monolayers in typically 250 ps. Wang, Jiang, and Steel ${ }^{18}$ reported also in GaAs QW's a 100-ps time for the hopping of localized excitons. They employed high-resolution frequency-resolved FWM.

Since the first experiments, the quality of the QW's fabricated has improved to such a degree that REE in the highenergy wing of the exciton resonance are considered to be free and should have large $L_{c}$ and exhibit pure superradiance. The question arises: what are the transport properties of these superradiant excitons? Using the observed superradiant lifetimes and the maximum velocity of REE in ideal GaAs SQW's of $10^{4} \mathrm{~m} / \mathrm{s}$, we can directly estimate a traveled path within its lifetime of $10^{-7} \mathrm{~m}$, which is smaller than $\lambda$. This calculation shows that REE are immobile but free. Of course the transport properties of the superradiant excitons may be affected by scattering, e.g., exciton-exciton or excitonphonon interaction. The latter is shown to be important at elevated temperatures, brings the exciton to a state with high momentum, and thus makes the exciton mobile. The role of exciton-exciton interaction in transport of REE is not yet studied and is one of the main issues addressed in the present paper.

In this paper we present ultrafast pump-probe measurements on the lifetime, transport and dephasing of REE in GaAs SQW's. To study the superradiant lifetime and the transport properties, we rely on a combination of two experimental techniques: transient reflection (TR) and photon echo (PE). In TR experiments, excitons are probed in a remarkably sensitive way, namely via the screening they achieve of the surface electric field. We show that excitons created in the high-wing of the resonance at low temperature and low density, decay superradiantly, may be considered as a free coherent state, and indeed are effectively immobile. For elevated free-exciton densities $N_{\mathrm{ex}}>10^{9} \mathrm{~cm}^{-2}$ exciton-exciton interaction is shown to produce transport.

This paper is organized as follows. In Sec. II we describe the samples and the experimental technique. We continue by introducing the mechanism allowing us to study energy relaxation and transport of REE. In Sec. III we present the experimental data and discuss the results, and in Sec. IV we conclude the paper.

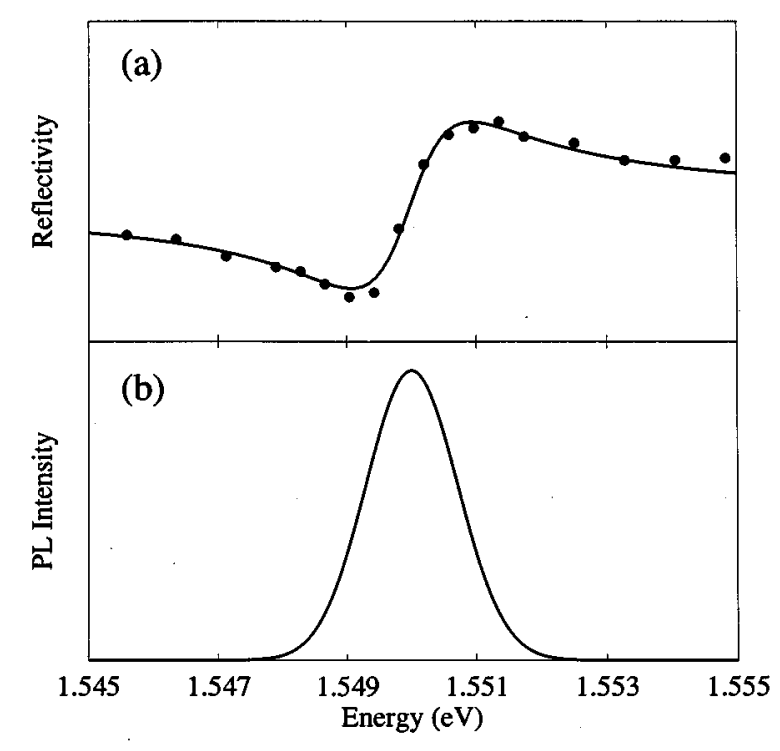

FIG. 1. cw reflection spectrum (upper trace) and luminescence spectrum (lower trace) in the $100-\AA \mathrm{SQW}$ at $5 \mathrm{~K}$. The solid curve in the upper panel is a calculation, explained in the text.

\section{EXPERIMENTAL METHODS}

\section{A. Samples}

We used GaAs SQW's fabricated on top of a semiinsulating chromium-doped (001) GaAs wafer. An intrinsic GaAs buffer layer of $1 \mu \mathrm{m}$ is deposited on the substrate, followed by a $60-\mathrm{nm} \mathrm{Al}_{x} \mathrm{Ga}_{1-x}$ As barrier $(x=0.27 \pm 0.01)$, a thin GaAs layer, forming the quantum well, a $300-\mathrm{nm}$ $\mathrm{Al}_{x} \mathrm{Ga}_{1-x} \mathrm{As}$ barrier, and a 30-nm GaAs cap layer. The samples are nominally undoped and molecular beam epitaxy grown at a substrate temperature of $580{ }^{\circ} \mathrm{C}$. The quantumwell thicknesses used in the experiments are 35, 50, 100, and $200 \AA$ A. The full width at half maximum linewidths of the $1 s-H H$ exciton resonances are determined by photoluminescence, and amount to $5.8,3.4,1.2$, and $0.6 \mathrm{meV}$, respectively. The reflection spectra all show a distinct dispersive curve around the $1 s-H H$ exciton absorption. Spectra are given in Fig. 1 for the 100- $\AA$ SQW. The Stokes shift turns out to be smaller than $1 \mathrm{meV}$ for all samples, as judged from comparing the $\mathrm{cw}$ reflection and photoexcitation spectrum (not shown) with the luminescence. The immeasurably small Stokes shift demonstrates the high quality of the samples.

\section{B. Pump-probe setup}

The experimental scheme is shown in Fig. 2. We use an argon-ion laser-pumped Ti:sapphire laser, producing a beam consisting of a 76-MHz Fourier-limited train of pulses that is split into two by a beam splitter. One of the beams is modulated at $100 \mathrm{kHz}$ by passing a fast mechanical chopper. The other beam meets a scanning optical delay line, a pneumatic shaker (Mannesmann-Rexroth Pneumatic KA-20 HUB) that runs at a velocity of $0.1 \mathrm{~m} / \mathrm{s}$ with a maximum delay of $0.7 \mathrm{~ns}$. Both beams are focused to an area of about $(100 \mu \mathrm{m})^{2}$ on the sample, under a mutual angle $\theta \approx 8^{\circ}$. Different colors for the pump and probe can be used starting with femtosecond pulses and filtering by insertion of a Fabry-Perot interferometer (FPI) in the pump arm and a single grating monochro- 


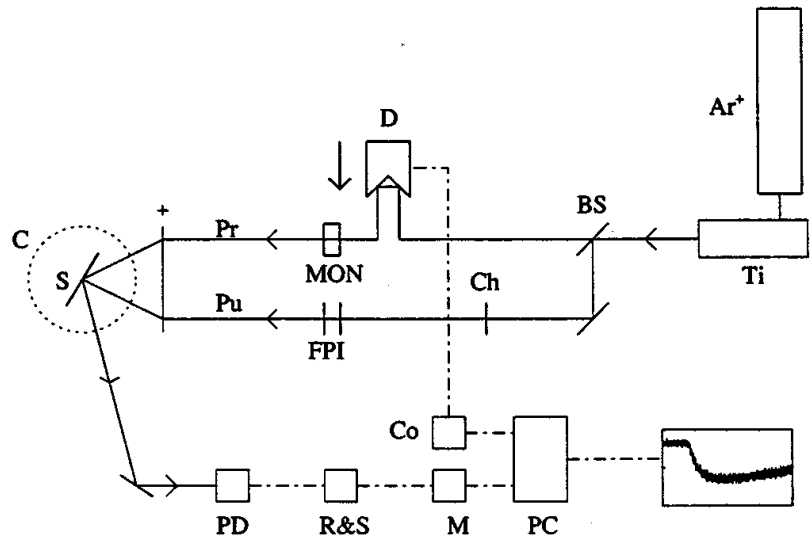

FIG. 2. Setup for the pump-probe experiments in the TR geometry. $\mathrm{Ar}^{+}$—argon-ion laser; $\mathrm{Ti}$ - Ti:sapphire laser; BS—beam splitter; $\mathrm{Ch}$-chopper; FPI-Fabry-Perot interferometer; MONmonochromator; $D$-delay line; $\mathrm{Pu}$ - pump beam; $\mathrm{Pr}$ - probe beam; $C$-cryostat; $S$-sample; PD—avalanche photodiode; R\&SRhode \& Schwartz test receiver; $M-\mathrm{HP}$ multimeter; Cocontroller delay line; PO-personal computer.

mator in the probe arm. The FPI has a free spectral range of $7.3 \mathrm{THz}$ and a finesse of about 30 . The monochromator has a resolution of $0.5 \mathrm{meV}$. The crosscorrelation of the two pulses is typically $2.5 \mathrm{ps}$. The pump and probe color can be chosen independently within the laser linewidth $(11 \mathrm{meV})$. Since pump and probe are derived from the same laser, the relative timing of the pulse trains is essentially jitter free. Alternatively we used the laser in the picosecond mode $(1.5 \mathrm{ps})$ for PE experiments and TR with one-color pump and probe beams. The intensity of the probe beam is taken at least a factor of 100 smaller than the pump, but in PE experiments pump and probe intensities are equal.

We have chosen the reflection geometry to avoid etching of the substrate and strain problems. In TR the probe beam, and, in PE, light emitted in the direction $2 \theta$, are led to a Peltier-cooled Hamamatsu C4777 photodiode. The bandwidth of the photodiode is $100 \mathrm{MHz}$, the sensitivity 2.5 $\mathrm{kV} / \mathrm{W}$, corresponding to 70 electrons per photon, and the background noise $5 \mathrm{pA} / \sqrt{\mathrm{Hz}}$. The performance of this photodiode approaches that of a photomultiplier tube. We demodulate the photocurrent at $100 \mathrm{kHz}$ over a $10-\mathrm{kHz}$ bandwidth, using a Rhode and Schwartz test receiver. The signal is read out every $10-\mu \mathrm{m}$ displacement of the delay line by a HP3458A voltmeter, and stored in a personal computer. Signal averaging is performed by repetitively scanning the delay line. The sample is held in an optical flow cryostat that is temperature controlled from $2 \mathrm{~K}$ upwards. Experiments are performed with equal horizontal polarization of the pump and probe. We checked that the results are identical for cross-polarized pump and probe in TR experiments.

\section{Optically induced blueshift of the exciton resonance}

Figure 3 shows examples of the signal for one-color pump and probe TR experiments. Curve 2 is taken during additional illumination with a $\mathrm{cw}$ halogen lamp $\left(0.5 \mathrm{~mW} / \mathrm{cm}^{2}\right)$, and curve 1 without the lamp. The signal amplitude significantly diminishes by the illumination, but the shape remains the same. We measure a signal in the case of pumping and

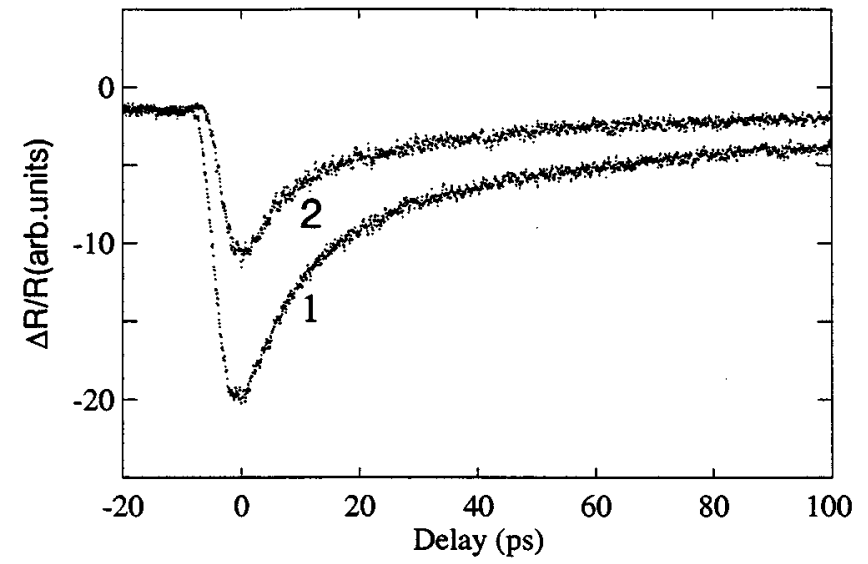

FIG. 3. TR trace in the 100 - $\AA \mathrm{SQW}$ at $5 \mathrm{~K}$ without (curve 1) and with cw illumination (curve 2).

probing resonantly with the exciton line, but no signal when either pump or probe is tuned out of resonance. The TR signal appears to be negative near the line center, and is positive but small at the wings. The observed change in amplitude suggests the involvement of screening of static electric fields by photocarriers.

To explain the signal we approximate 2D excitons by a sheet of Lorentz oscillators located at a distance $d$ from the sample surface, and the rest of the sample as a stack of layers with the appropriate dielectric constants. In a standard matrix calculation we computed the amplitude of the reflected waves, normalized to the amplitude of the incident wave both traveling perpendicularly to the sheet. The result is shown in the upper panel of Fig. 1 as a solid curve that tracks the measured cw-reflection spectrum (full dots) well. Subsequently we calculated the dynamical reflection spectrum for the case of a transient broadening, shift, or decrease in magnitude of the resonance (not shown). A comparison of these spectra to the measured signals leads to the conclusion that the TR signals in our samples correspond to a transient blueshift of the exciton line induced by the pump pulse.

The origin of this blueshift is screening of the surface electric field by excitons produced by the pump. An electric field is generally present near the surface, due to Fermi-level pinning at the surface, and penetrates the QW. For a semiinsulating substrate, surface field strengths of $E_{0}$ $=10^{5}-10^{6} \mathrm{~V} / \mathrm{m}$ are expected. ${ }^{19}$ The exciton resonance then shifts by the action of the quantum-confined Stark effect (QCSE). ${ }^{20-24}$ We estimate the screening field of $N$ excitons per unit of surface by

$$
\Delta E=\frac{N_{e}}{4 \pi \epsilon_{0} \epsilon_{r}}
$$

with $e$ the elementary charge and $\epsilon_{0} \epsilon_{r}$ the dielectric constant. For exciton densities of $10^{9} \mathrm{~cm}^{-2}, \Delta E=1.1$ $\times 10^{3} \mathrm{~V} / \mathrm{m}$. Using the result of Miller et al. ${ }^{19}$ for the coefficient $\alpha$ of the the QCSE, $\alpha=1.7 \times 10^{-11} \mathrm{~m}^{2} \mathrm{meV} / \mathrm{V}^{2}$, we arrive at a blueshift $2 \alpha \Delta E E_{0}$, equal to $4 \mu \mathrm{eV}$ for $E_{0}$ $=10^{5} \mathrm{~V} / \mathrm{m}$. According to our calculations this value corresponds to a change of reflection of $2 \times 10^{-5}$, within our detection sensitivity. 
Our observations are consistent with photoreflectance modulation described in Ref. 25. In these experiments an exciton line shift was found to be induced by excitons that could clearly be related to screening of the surface electric field and the QCSE. We conclude that the surface electric field in our sample is instrumental for generating a TR signal.

The TR technique may be used not only to study the decay but also the spatial transport of REE in an inhomogeneously broadened exciton resonance. A spectrally narrow pump frequency $\hbar \omega_{1}$, for example, creates a spatial distribution of excitons

$$
\eta_{0}(\mathbf{r}) \propto I_{1} f\left(\omega_{1}, \mathbf{r}\right),
$$

where $I_{1}$ is the intensity of the pump beam and $f(\omega, \mathbf{r})$ is the homogeneous absorption line profile at position $\mathbf{r}$ in the quantum well. Taking for $f(\omega, \mathbf{r})$ a Lorentzian of width $\Gamma_{h}$ with a local energy $\hbar \omega_{r}$, and an amplitude distributed according to a Gaussian of width $\Gamma_{i}$ centered around $\hbar \omega_{0}$, we obtain

$$
f\left(\omega_{1}, \mathbf{r}\right) \propto \frac{\exp \left[-4 \hbar^{2}\left(\omega_{\mathbf{r}}-\omega_{0}\right)^{2} / \Gamma_{i}^{2}\right]}{\left[\hbar^{2}\left(\omega_{1}-\omega_{\mathbf{r}}\right)^{2}+\Gamma_{h}^{2}\right]} .
$$

Equations (5) and (6) tell us that there will be a significant exciton population created only if the local resonance energy $\hbar \omega_{\mathbf{r}}$ is sufficiently close to $\hbar \omega_{1}$, i.e., within $\Gamma_{h}$. The suffix $\mathbf{r}$ of $\omega_{\mathbf{r}}$ is to express that the exciton resonance frequency is position dependent. Thus spectral diffusion reflects spatial transport. In a TR experiment, with pump and probe at the same laser frequency $\omega_{1}$, we create excitons at positions given by Eq. (6), leading to a local and temporal blueshift of the exciton resonance as explained above. Consequently, the TR signal will drop as soon as excitons either decay at the location of creation or escape to other regions of the QW. In TR experiments with different pump and probe frequencies, we may probe the transport directly.

\section{RESULTS AND DISCUSSION}

\section{A. Low exciton density and temperature, superradiance}

In this section we present the results of TR and PE experiments in SQW's for low exciton densities $\left(N_{\mathrm{ex}} \sim 10^{9} \mathrm{~cm}^{-2}\right)$ and temperature $(T=5 \mathrm{~K})$. Figure 4 shows a selection of TR signals for equal pump and probe frequencies in the $50-\AA \mathrm{SQW}$ at the spectral positions as indicated in the inset, the luminescence spectrum. The solid lines are fits to a uniexponential function convoluted with the instrumental profile. The decay constant $T_{1}$ decreases from 26 to $7 \mathrm{ps}$ for increasing photon energy. Similar behavior is found for the other quantum wells: In the $35-\AA \mathrm{SQW}$, the lifetime goes from 17.9 to $5.2 \mathrm{ps}$, and in the $100-\AA \mathrm{SQW}$ from 15 to $8 \mathrm{ps}$. For the 200- $\AA$ SQW, the inhomogeneous broadening is smaller than the laser linewidth and $T_{1}=13 \mathrm{ps}$. The decay times, taken at the high-energy side of the exciton resonance, namely $5.2,7,8$, and $13 \mathrm{ps}$, apparently increase with $L_{z}$.

The decay of the signal presented in Fig. 4 measures loss of excitons in the region of creation. We show that under the present conditions exciton transport is absent, using results of the one- and two-color TR experiments described now. In Fig. 5 we present the one-color TR signal, curves labeled 1,

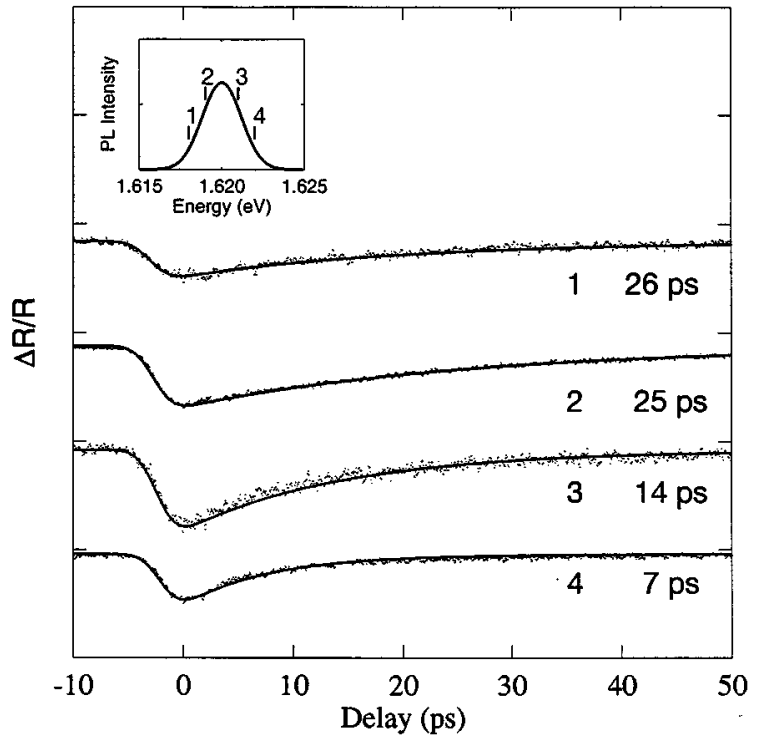

FIG. 4. TR for the $50-\AA \mathrm{SQW}$ at $T=5 \mathrm{~K}$ and an exciton density of $10^{9} \mathrm{~cm}^{-2}$. Photon energies are indicated in the inset, which shows the PL spectrum. Full lines are fits discussed in the text.

taken in the $100-\AA$ well $1 \mathrm{meV}$ above line center at the indicated energy 1 in the inset. Curves 2 are the two-color measurements when pumping at the same energy as curve 1, but probing at an energy $1.5 \mathrm{meV}$ lower in the line, indicated in the inset. Clearly, no signal is observed for low densities, Fig. 5(a), in contrast to the case of high densities, Fig. 5(b). Quite remarkably, the amplitude of curve 2 in Fig. 5(b) is much larger than that of curve 1 in Fig. 5(b), signifying exciton transport at high densities. The high-density data are

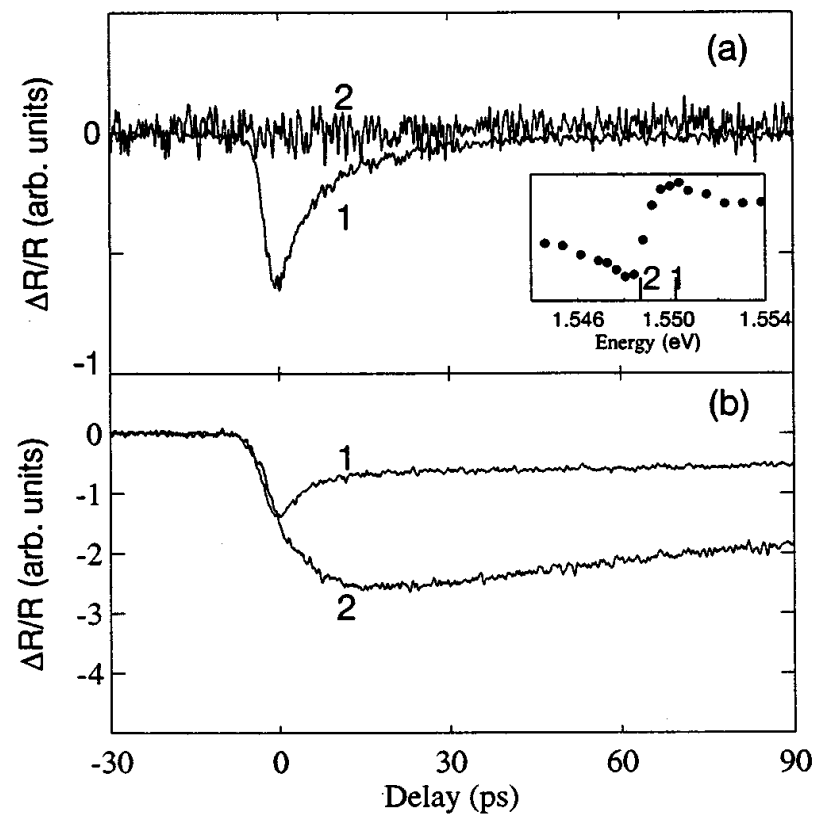

FIG. 5. TR signal in the $100-\AA \mathrm{SQW}$ at $5 \mathrm{~K}$, with pump and probe $1 \mathrm{meV}$ above line center (curve 1), and TR signal with pump $1 \mathrm{meV}$ above line center and probe $0.5 \mathrm{meV}$ below line center (curve 2), for (a) $10^{9}$ excitons $\mathrm{cm}^{-2}$ and (b) 5 $\times 10^{9}$ excitons $\mathrm{cm}^{-2}$. 
further discussed in Sec. III B. At this point we can already conclude that exciton transport is virtually absent at low densities and temperature.

Now we compare $T_{1}$ with the dephasing time $T_{2}$ measured by PE, under the same conditions. We fit a uniexponential curve to our data, with the time constant $\tau$ convoluted with a Gaussian line to account for the instrumental resolution. The polarization dephasing time is computed by taking $T_{2}=4 \tau$, the appropriate expression for inhomogeneously broadened resonances. High in the resonance we then find 12 ps in the 50- and 100- $\AA$ SQW's and 8 ps in the $35-\AA$ SQW (data not shown).

Lower in the resonance, however, $T_{2}$ is larger, an effect reported earlier by Hegarty and Sturge, ${ }^{26}$ and attributed to localization: For the 50- $\AA \mathrm{SQW}$, we observe $T_{2}=25 \mathrm{ps}$. For the $35-\AA \mathrm{SQW}$ the elongation is even more pronounced. For the $100-\AA$ SQW, which has the smallest inhomogeneous width, however, the effect is quite small, namely, an increase from 12 to 14 ps.

Polarization dephasing generally is caused by $T_{1}$ and $T_{2}^{\prime}$,

$$
1 / T_{2}=1 / 2 T_{1}+1 / T_{2}^{\prime} .
$$

From the measured $T_{2}$ and $T_{1}$, we may compute $T_{2}^{\prime}$ using Eq. (7). For the 100- $\AA$ SQW, $1 \mathrm{meV}$ above line center, we find a mean value for $T_{2}^{\prime}=48 \mathrm{ps}$, with a lower limit of $26 \mathrm{ps}$, in any case much longer than $T_{1} \approx 8 \mathrm{ps}$. The large pure dephasing time is also found for the 50- $\AA \mathrm{SQW}$ high in the resonance. This important result shows that here $T_{2}$ is governed by energy relaxation,

$$
1 / T_{2} \approx 1 / 2 T_{1} .
$$

Since nonradiative recombination of excitons is known to be negligibly small, and transport is absent, we conclude that the $T_{1}$ 's measured high in the line in the 50- and $100-\AA$ SQW's are the superradiant lifetimes of free excitons. Apparently the exciton superradiates rather than collides elastically. Hanamura ${ }^{7}$ pointed out that this remarkable circumstance should be present for free excitons in high-quality SQW's. Knowing $T_{2}^{\prime}$ and using Eq. (2), we estimate the coherence length $L_{c}$, and find, for the 100- $\AA$ SQW, $L_{c}$ $=190 \mathrm{~nm}$, with a lower limit of $100 \mathrm{~nm}$. This value is close to $\lambda$, implying that the measured radiative lifetime of the excitons virtually represents the superradiant lifetime of Eq. (1). Indeed, $T_{1}=8 \mathrm{ps}$ is approximately the predicted value of $\tau_{0}=10 \mathrm{ps}$.

In the low-energy wing of the exciton resonance, in the $100-\AA \mathrm{SQW}$ we find $T_{1}=15 \mathrm{ps}$, longer than high in the line. This points to a decreased value of $L_{c}$, in this case $80 \mathrm{~nm}$ according to Eq. (3). This value is larger than the $L_{c}$ computed from Eq. (2), valid for free excitons. All SQW data taken in the low-energy parts of the line exhibit the same discrepancy. From this we conclude that the free-exciton picture fails, and that excitons localize in the low-energy wing, in agreement with earlier findings. ${ }^{18,26}$

\section{B. High $N_{\mathrm{ex}}$ and $T$, transport and dephasing}

By increasing the excitation density $N_{\mathrm{ex}}$ and temperature $T$, we can study the dephasing and transport properties of REE in the presence of interaction. As already discussed in

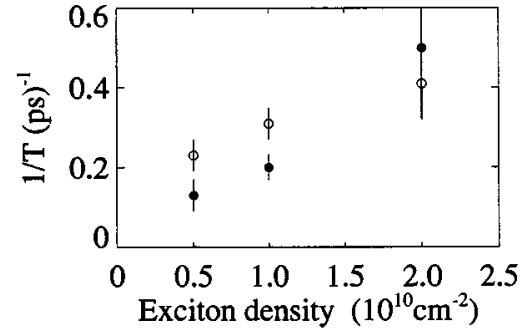

FIG. 6. Decay rate $T_{1}^{-1}$ of TR signal pumping and probing the free excitons $(\bigcirc)$ and reciprocal rise time of TR signal when pumping free excitons and probing localized excitons $(\mathbf{O})$ as a function of exciton density for the 100-A SQW.

Sec. III A, no TR signal is detectable at low densities, at least when the pump and probe energies differ by more than 1 meV [Fig. 5(a)]. At exciton densities above $2 \times 10^{9} \mathrm{~cm}^{-2}$, however, we measure a clear TR signal in two-color experiments, which is a direct proof of exciton transport [Fig. $5(\mathrm{~b})]$.

TR data for $N_{\mathrm{ex}}=10^{10} \mathrm{~cm}^{-2}$ and $T=5 \mathrm{~K}$ in the $100-\AA$ SQW are presented in Fig. 5(b). As stated above, curve 1 corresponds to free excitons and equal pump and probe energies. Curve 2 is obtained when pumping free excitons and probing the localized states (see the inset). Curve 2 reveals a rise taking $7 \mathrm{ps}$, followed by a delay of hundreds of picoseconds. Within the experimental resolution the rise time appears to be independent of the frequency difference between pump and probe. The rise time does, however, depend on exciton density (see Fig. 6, full dots). As demonstrated in Sec. II C, one-color TR experiments monitor local decay by superradiance and escape of excitons, while two-color TR experiments probe exciton transport. The open dots in Fig. 6 are the measured decay rates of the one-color TR signals, $T_{1}^{-1}$ vs $N_{\text {ex }}$. A simple rate-equation analysis reveals that the rise time should be equal to $T_{1}$, as observed. Inspection of the amplitudes of the two-color signals and comparison to the results of the rate-equation analysis, lead to the conclusion that the speeding up of $T_{1}^{-1}$ for increased exciton densities is exclusively due to spatial escape, induced by exciton-exciton collisions.

In order to examine the effects of exciton-exciton collisions on $T_{2}$, we compare PE and TR experiments in the 100- $\AA$ SQW again on free excitons in Fig. 7. The observed rise of $T_{2}^{-1}$ with $N_{\text {ex }}$ (open dots in Fig. 7) is known to be produced by exciton-exciton collisions. ${ }^{27}$ We find for free excitons in the $100-\AA$ SQW $T_{2}^{-1}=7.0 \times 10^{10} \mathrm{~s}^{-1}$ $+10.0 N_{\mathrm{ex}} \mathrm{s}^{-1} \mathrm{~cm}^{2}$ (full line tracking the PE data in Fig. 7). These values are in good agreement with earlier measurements. ${ }^{27}$ Quite remarkably, multiplication of this line for $T_{2}^{-1}$ by a factor of 2 yields the line through the $T_{1}^{-1}$ data (full dots in Fig. 7), i.e., $T_{2} \approx 2 T_{1}$, over the measured range of $N_{\text {ex }}$. We conclude from this that in inhomogeneously broadened resonances any exciton-exciton collision produces transport.

We now turn to the question how exciton-exciton scattering may induce transport of superradiant excitons in the absence of thermal phonons. We consider interaction between two excitons in the inhomogeneously broadened exciton resonance. In an exciton-exciton collision process energy 


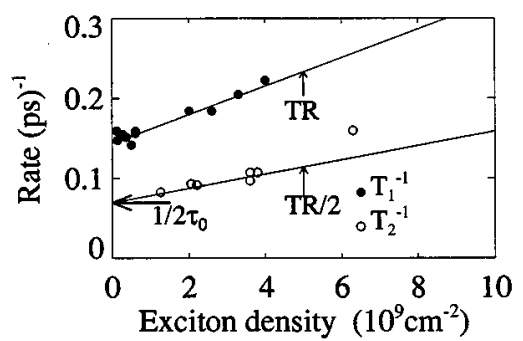

FIG. 7. Fast decay rate in TR $(\bigcirc)$ and $\mathrm{PE}(\mathrm{O})$ vs exciton density for the $100-\AA \mathrm{SQW}$ at $5 \mathrm{~K}$. PE data merge into TR data when multiplying by a factor of 2 . Indicated with an arrow is $1 / 2 \tau_{0}$, as deduced from TR data, at low densities.

conservation is now possible, when the potential energy of one exciton is exchanged for the kinetic energy of the other. In a two-exciton collision a $\mathbf{q} \approx 0$ exciton may then gain kinetic energy at the expense of another exciton. The increase in kinetic energy is typically the inhomogeneous linewidth. Such collisions inherently involve transport, as observed. In a QW with disorder, momentum conservation does not hold if at least one of the excitons involved in the collision is localized. A more detailed model of exciton-exciton interaction in disordered QW's is required.

The situation markedly changes when localized excitons are excited, low in the line. These results are shown in Fig. 8. Curve 1 is TR data with equal pump and probe energies, and curve 2 corresponds to probing high in the line, both at $5 \mathrm{~K}$. As for curve 2, free excitons are probed in curve 3, but now at $100 \mathrm{~K}$ instead of $5 \mathrm{~K}$. We kept track of the shifting of the band gap to lower energies by properly tuning the pump and probe frequencies. The rise time of the signal when probing at higher energies than the pump amounts to $13 \mathrm{ps}$ at $5 \mathrm{~K}$, with a greatly diminished amplitude compared to curve 1 .

Figure 9 shows the density dependence of $T_{1}^{-1}$ (open circles) and $T_{2}^{-1}$ (closed circles) at $5 \mathrm{~K}$ when pumping the

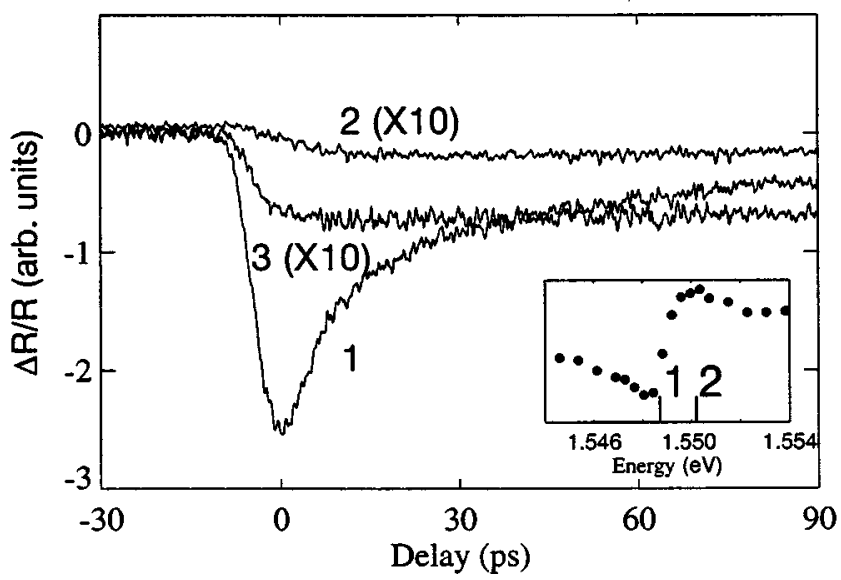

FIG. 8. TR data in the $100-\AA$ SQW at an exciton density of 5 $\times 10^{9} \mathrm{~cm}^{-2}$ when pumping the localized states. The probe is resonant with the localized states in curve 1 , and the free states in curve 1. Curves 1 and 2 are taken at $5 \mathrm{~K}$, and curve 3 at $100 \mathrm{~K}$. The pump and probe wavelength in curve 3 have been properly tuned to compensate for the shift in band gap at elevated temperature. Pump and probe energies are indicated in the inset, the cw-reflection spectrum at $5 \mathrm{~K}$.

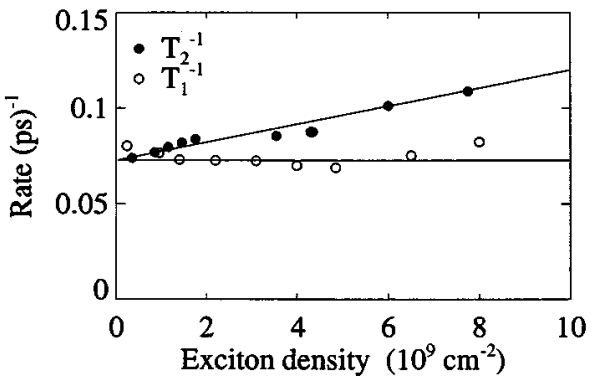

FIG. 9. TR data $(\bigcirc)$ and PE $(\mathbf{O})$, vs pump power for the $100-\AA$ SQW taken at $5 \mathrm{~K}$, when pumping the localized states.

localized excitons. The time $T_{1}$ is, within errors, independent of the exciton density. However, the dephasing rate $T_{2}^{-1}$ increases with $N_{\text {ex }}$, but slower than for the case of free excitons. Apparently, exciton-exciton scattering induces dephasing for localized excitons in a similar way as it does for free excitons, but does not affect $T_{1}$, in contrast to the freeexciton case. Hence for localized excitons the transport induced by exciton-exciton interaction is strongly suppressed in comparison to the free-exciton case. This is consistent with TR signals for pumping in the localized part and probing higher. We note that the amplitude of curve 2 in Fig. 8 is two orders of magnitude smaller than curve 1.

For elevated temperatures the rise time decreases (compare curves 2 and 3 of Fig. 8), but the amplitude increases. Obviously exciton-phonon interaction also affects the transport properties of the superradiant excitons, since the TR signal increases with rising temperature (curve 3, Fig. 8). The amplitude of this curve is much larger than the amplitude of curve 2 of Fig. 8 measured at $5 \mathrm{~K}$ under otherwise the same conditions.

The rise time of the TR signal, when pumping localized states and probing the free exciton state, has the same value and temperature dependence as the decay time $T_{1}$ of TR when pumping and probing the localized excitons (see Fig. 10). The solid curve is a fit to a thermally activated behavior. For localized excitons the thermally activated behavior of $T_{1}$ and $T_{2}$ that we observed appeared to be consistent with the results of Hegarty, Goldner, and Sturge, ${ }^{15}$ and thus is not discussed in detail in the present paper.

Finally we discuss the long decay of the TR signals, observed at high $N_{\text {ex }}$ or $T$, produced by the excitons after many exciton-exciton and exciton-phonon collisions. We observe a

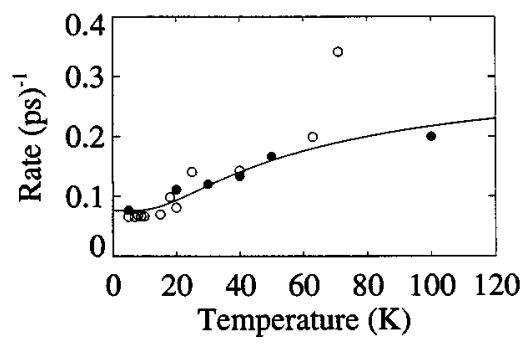

FIG. 10. (-) Reciprocal rise time of the TR signal vs temperature for pumping localized excitons in a $100-\AA$ SQW and probing free excitons. Fast decay rate $(O)$ TR vs temperature when pumping and probing localized excitons for $N_{\mathrm{ex}}=10^{10} \mathrm{~cm}^{-2}$. The curve is discussed in Sec. III B. 
decay time of a couple of hundred picoseconds that is independent of pump and probe energies, in agreement with the luminescence decay time of nonresonantly excited excitons. ${ }^{28,29}$ Indeed, in case of high exciton densities or temperature the excitons are expected to form a statistical distribution over the available states similar to the one present for the nonresonantly excited excitons, already extensively studied. In the limit of high density or temperature the excitons produce a Boltzmann distribution which no longer reflects the specific initial excitation conditions.

\section{CONCLUSION}

To summarize, we obtain insight into the transport properties of $2 \mathrm{D}$ superradiant $1 s-H H$ excitons in inhomogeneously broadened resonances employing transient reflection and photon-echo techniques.

High in the resonance, and for low density and temperature, the superradiant lifetimes are determined to be 5, 7, 8, and 13 ps for 35-, 50-, 100-, and 200- $\AA$ SQW's, respectively. We show that the polarization decay is completely governed by energy relaxation, leading to an estimate of the typical coherence length for these free excitons of $\sim 200 \mathrm{~nm}$. Exciton transport is shown to be slow in comparison to the radiative decay, confirming the expectation that the resonantly excited excitons high in the resonance are free but immobile.
Localization effects low in the exciton resonance give rise to a decrease in coherence length, an increase in radiative lifetime, and significant pure dephasing.

Exciton-exciton and exciton-phonon collisions are shown to induce transport between areas of different thicknesses in the quantum well. The increase of the dephasing rate of free excitons in the presence of exciton-exciton collisions is demonstrated to be exclusively due to the speeding up of this transport, and not induced by pure dephasing. For localized states, we demonstrate that only a small fraction is mobile under conditions of strong optical pumping at low temperatures, that pure dephasing by exciton-exciton collisions does play a role, and that thermal activation processes induce transport at higher temperature.

The transport of the free $\mathbf{q} \approx 0$ excitons in inhomogeneously broadened resonances at low temperature is possible thanks to the exchange of potential and kinetic energies in an exciton-exciton collision. A detailed model, however, is lacking.

\section{ACKNOWLEDGMENTS}

We thank F. J. M. Wollenberg and R. Beelen for technical assistance. A.V.A. gratefully acknowledges INTAS (94-395) for financial support. This work was sponsored by the Dutch science foundations FOM and NWO.
*Permanent address: A. F. Ioffe Physical-Technical Institute, 194021 St. Petersburg, Russia.

${ }^{1}$ R. H. Dicke, Phys. Rev. 93, 99 (1954).

${ }^{2}$ S. de Boer and D. A. Wiersma, Chem. Phys. Lett. 165, 45 (1990).

${ }^{3}$ J. Grad, G. Hernandez, and S. Mukamel, Phys. Rev. A 37, 3835 (1988).

${ }^{4}$ V. M. Agranovich and O. A. Dubovskii, Pis'ma Zh. Eksp. Teor. Fiz. 3, 345 (1966) [JETP Lett. 3, 223 (1966)].

${ }^{5}$ K. C. Liu, Y. C. Lee, and Y. Shan, Phys. Rev. B 11, 978 (1975).

${ }^{6}$ K. C. Liu and Y. C. Lee, Physica A 102, 131 (1980).

${ }^{7}$ E. Hanamura, Phys. Rev. B 38, 1228 (1988).

${ }^{8}$ L. C. Andreani, F. Tassone, and F. Bassani, Solid State Commun. 77, 641 (1991).

${ }^{9}$ D. S. Citrin, Phys. Rev. B 47, 3832 (1993).

${ }^{10}$ E. Hanamura, in Optical Switching in Low-Dimensional Systems, edited by H. Haug and L. Banyai (Plenum, New York, 1989), p. 203.

${ }^{11}$ B. Deveaud, F. Clérot, N. Roy, K. Satzke, B. Sermage, and D. S. Katzer, Phys. Rev. Lett. 67, 2355 (1991).

${ }^{12}$ A. Vinatierri, J. Shah, T. C. Damen, D. S. Kim, L. N. Pfeiffer, M. Z. Maialle, and L. J. Sham, Phys. Rev. B 50, 10868 (1994).

${ }^{13}$ M. Hübner, J. Kuhl, T. Stroucken, A. Knorr, S. W. Koch, R. Hey, and K. Ploog, Phys. Rev. B 76, 4199 (1996).

${ }^{14}$ H. Wang, J. Shah, T. C. Damen, and L. N. Pfeiffer, Phys. Rev. Lett. 74, 3065 (1995)
${ }^{15}$ J. Hegarty, L. Goldner, and M. D. Sturge, Phys. Rev. B 30, 7346 (1984).

${ }^{16}$ H. Hillmer, A. Forchel, S. Hansmann, M. Morohashi, E. Lopez, H. P. Meier, and K. Ploog, Phys. Rev. B 39, 10901 (1989).

${ }^{17}$ B. Deveaud, T. C. Damen, J. Shah, and C. W. Tu, Appl. Phys. Lett. 51, 828 (1987).

${ }^{18}$ H. Wang, M. Jiang, and D. G. Steel, Phys. Rev. Lett. 65, 1255 (1990).

${ }^{19}$ I. Sela, D. E. Watkin, B. K. Laurich, D. L. Smith, S. Subbanna, and H. Kroemer, Phys. Rev. B 43, 11884 (1991).

${ }^{20}$ D. A. B. Miller, D. S. Chemla, T. C. Damen, A. C. Gossard, W. Wiegmann, T. H. Wood, and C. A. Burrus, Phys. Rev. B 32, 1043 (1985)

${ }^{21}$ G. Bastard, E. E. Mendez, L. L. Chang, and L. Esaki, Phys. Rev. B 28, 3241 (1983).

${ }^{22}$ E. J. Austin and M. Jaros, Phys. Rev. B 31, 5569 (1985).

${ }^{23}$ J. A. Brum and G. Bastard, Phys. Rev. B 31, 3893 (1985).

${ }^{24}$ M. Matsuura and T. Kamizato, Phys. Rev. B 33, 8385 (1986).

${ }^{25}$ B. V. Shanabrook, O. J. Glembocki, and W. T. Beard, Phys. Rev. B 35, 2540 (1987).

${ }^{26}$ J. Hegarty and M. D. Sturge, J. Opt. Soc. Am. B 2, 1143 (1985).

${ }^{27}$ L. Schultheis, J. Kuhl, A. Honold, and C. W. Tu, Phys. Rev. Lett. 57, 1635 (1986).

${ }^{28}$ J. Feldmann, G. Peter, E. O. Göbel, P. Dawson, K. Moore, C. Foxon, and R. J. Elliot, Phys. Rev. Lett. 59, 2337 (1987).

${ }^{29}$ M. Colloci, M. Gurioli, A. Vinatierri, F. Fermi, C. Deparis, J. Massies, and G. Neu, Europhys. Lett. 12, 417 (1990). 